environmental factors and the effect on infant growth and development at the postpartum 8th month.

Method As a part of the cohort study at Hacettepe University Department of Social Paediatrics, 74 breast milk samples and 46 infant hair samples were taken at the postpartum 8th month. Mothers' environmental exposure were evaluated by a semistructured questionnaire.

Results $\mathrm{Pb}, \mathrm{Hg}, \mathrm{Cd}$ were detected in breast milk samples of 91\%, 87\%, 99\%, respectively. Breast milk Hg levels were associated with maternal vitamin use within the first 2 months after delivery, breast milk Cd levels were higher in cases with expanded family, living in older house and in urban with air pollution during childhood. Higher infantile hair Cd levels were higher in cases with expanded family, living older house, maternal history of anaemia and postpartum anaemia. In girls $\mathrm{z}$ scores of birth weight, height for age at the $8^{\text {th }}$ month were inversely correlated with infant hair $\mathrm{Pb}$ levels. In boys, $\mathrm{z}$ scores of body mass index at the 8th month were inversely correlated with infant hair Cd levels. The levels of breast milk $\mathrm{Hg}$ levels were higher in infants with Denver II suspicious/abnormal.

Conclusion It is suggested that presence of $\mathrm{Pb}, \mathrm{Hg}, \mathrm{Cd}$ in breast milk and infant hair have been affected infants of developmental and growth parameters. Preventive measures must be taken to reduce environmental toxic metals pollution and efforts should be made for the management of anaemia in females in all age groups.

\section{PO-0374 HYPOMELANOSIS OF ITO - CASE REPORT}

${ }^{1}$ D Popescu, ${ }^{2} \mathrm{G}$ Florescu, ${ }^{1} \mathrm{~A}$ Brinzan, ${ }^{1} \mathrm{P}$ Calistru, ${ }^{3} \mathrm{C}$ Strugaru. ${ }^{1}$ Pediatrics, Dr V Babes Foundation, Bucharest, Romania; ${ }^{2}$ Dermathology, Dr V Babes Foundation, Bucharest, Romania; ${ }^{3}$ Genetics, Carol Davila University of Medicine and Pharmacy, Bucharest, Romania

\subsection{6/archdischild-2014-307384.1021}

Background The authors report a case of Hypomelanosis of Ito (HI), a rare neurocutaneous syndrome associated with musculoskeletal, neurological alteration and ophthalmologic abnormalities.

Methods A 9 month old girl was brought for evaluation of hypopigmented skin lesions and strabismus. Clinical examination revealed whirled hypochromic skin lesions only in the left side of the body (neck and trunk) following the lines of Blaschko, compatible with $\mathrm{HI}$ and hypotonia. She presents also: strabismus, genu valgus and pubis pilosity. She attained head control at 4 month and failure to sit without support.

Results The blood tests, hormonal tests, abdominal and cranial ultrasound were normal. Electroencephalogram revealed diffuse cortico-subcortical dysfunction. MRI of head was normal. Blood karyotyping showed mosaicism. Differential diagnosis: nevus depigmentosus, tuberous sclerosis, vitiligo. Treatment: no treatment for the cutaneous lesions. Appropriate specialty care for orthopaedic and visual problems.

Conclusions

1. Numerous complications have been associated with $\mathrm{HI}$ involving the central nervous system, musculoskeletal, ophthalmologic alteration and urogenital malformation.

2. Many chromosomal abnormalities have been reported in association with $\mathrm{HI}$.

3. The prognosis of $\mathrm{HI}$ is determined by de associated abnormalities.

\section{PO-0375 ORAL AND DENTAL FINDINGS IN MUCOPOLYSACCHARIDOSIS TYPE}

${ }^{1} \mathrm{~N}$ Boutrid, ${ }^{1} \mathrm{H}$ Rahmoune, ${ }^{1} \mathrm{~B}$ Bioud, ${ }^{2} \mathrm{FC}$ Rahmoune. ${ }^{1}$ Pediatrics, Child and Mother Hospital, Setif, Algeria; ${ }^{2}$ Anesthesiology Rescucitation, Bicêtre Hospital, Paris, France

\subsection{6/archdischild-2014-307384.1022}

Introduction Mucopolysaccharidosis type $\mathrm{I}$ is an autosomal recessive inborn error of metabolism characterised by accumulation of incompletely degraded glycosaminoglycans leading to systemic impairment.

Our report presents the oro-dental and radiographic findings in 06 patients with MPS I

Methods and description The examination of the Mucopolysaccharidosis type I affected children followed in our clinic reveals characteristic abnormalities such as hypoplastic peg-shaped teeth and dysplastic teeth and gingival hyperplasia.

In all our patients, dental panoramic radiograph underlines these aspects.

Results and discussion The oral and dental findings of MPS I include hyperplastic gingiva, macroglossia, high-arched palate, short mandibular rami with abnormal condyles, spaced hypoplastic peg-shaped teeth with retarded eruption; and localised dentigerous cyst-like radiolucencies

Guven et al.(Jan 2008) have investigated the ultra structural and chemical properties of MPS I (Hurler) teeth. The dentin of the primary teeth was characterised by extremely narrow dentinal tubules with an irregular wave-like pattern. The enamel-dentin junction was poorly shaped, micro gaps occurred and the enamel displayed an irregular arrangement of prisms. The enamel and the dentin had an abnormal protein structure and the dentin protein content was low.

The mucopolysaccharidoses (MPS) are prominent among the lysosomal storage diseases. The intra-lysosomal accumulation of glycosaminoglycans (GAGs) in this group of diseases induces a cascade of responses affecting cellular functions and maintenance of the extra-cellular matrix.

Conclusion As well as skeletal problems, mucopolysaccharidoses' patients have dental with specific deformities.

Teeth involvement is highlighted, having an eye to the possibilities of reversing these oro-dental changes with enzyme replacement therapy.

\section{PO-0376 A NOVEL CASE OF UNBALANCED TRANSLOCATION OF CHROMOSOME 3 AND 7}

${ }^{1} \mathrm{~S}$ Rao, ${ }^{1} \mathrm{E}$ Hussien, ${ }^{2} \mathrm{M}$ Lees, ${ }^{1} \mathrm{~A}$ Shastri. ${ }^{1}$ Paediatrics and Neonatology, Colchester Hospital University NHS Foundation Trust, Colchester, UK; ${ }^{2}$ Clinical Genetics, Great Ormond Street Hospital for Children NHS Foundation Trust, London, UK

\subsection{6/archdischild-2014-307384.1023}

Background It is estimated that 1 in 500 to 1 in 625 human newborns have a balanced reciprocal chromosomal translocations. Such individuals are usually healthy and do not have any specific features. We report an unusual case of unbalanced translocation of chromosome 3 and 7 and describe its features.

Casereport A female baby born at 37 weeks gestation by a spontaneous vaginal delivery was admitted to our NICU with poor Apgar scores. Immediately after birth, she had a poor ventilatory effort requiring continuous positive airway pressure (CPAP) and further deterioration in her respiratory function led to intubation andsurfactant administration. Echocardiogram revealed small perimembranous VSD. She had dysmorphic features including 